\title{
Evaluation of a microcolony growth monitoring method for the rapid determination of ethambutol resistance in Mycobacterium tuberculosis
}

\author{
Alice L den Hertog ${ }^{1 *}$, Sandra Menting ${ }^{1}$, Ernst T Smienk ${ }^{1}$, Jim Werngren ${ }^{2}$, Sven Hoffner ${ }^{2}$ and Richard M Anthony ${ }^{1}$
}

\begin{abstract}
Background: Due to the increasing prevalence of Mycobacterium tuberculosis strains resistant to one or more antibiotics, there is a need for new quantitative culture methods both for drug susceptibility testing and for validation of mutations putatively associated with drug resistance. We previously developed a (myco) bacterial culture method, in which multiple growing microcolonies are monitored individually. Transfer of the growing microcolonies to selective medium allows the effect on the growth rate of each individual colony to be determined. As entire growing colonies are exposed to antibiotics rather than re-subbed, a second lag phase is avoided and results are obtained more rapidly. Here we investigate the performance of the microcolony method to differentiate between ethambutol (EMB) resistant, intermediate and susceptible strains.
\end{abstract}

Methods: One week old microcolonies from a reference panel of four strains with known EMB susceptibility were transferred to different concentrations of EMB. Growth rates during the $1^{\text {st }} 2$ days of exposure were used to set up classification criteria to test and classify a blinded panel of 20 tuberculosis strains with different susceptibilities.

Results: For 18 strains (90\%) reference culture results corresponded to our classifications based on data collected within 9 days of inoculation. A single strain was classified as Intermediate instead of Susceptible, and 1 strain could not be classified due to a contamination.

Conclusions: Using a microcolony growth monitoring method we were able to classify, within 9 days after inoculation, a panel of strains as EMB susceptible, intermediate or resistant with $90 \%$ correlation to the reference methods.

Keywords: Ethambutol, Drug susceptibility testing, Mycobacterium tuberculosis, Culture

\section{Background}

The increasing prevalence of Mycobacterium tuberculosis strains resistant to one or more antibiotics makes drug susceptibility testing (DST) a high priority. The rapid molecular detection of resistance e.g. for rifampicin (RIF) by automated molecular methods [1] creates an additional demand for phenotypic susceptibility testing to other agents. In fact a complete prediction of the in vivo susceptibility of strains to available drugs is

\footnotetext{
* Correspondence: a.d.hertog@kit.nl

'Royal Tropical Institute, KIT Biomedical Research, Meibergdreef 39, 1105, AZ, Amsterdam, The Netherlands

Full list of author information is available at the end of the article
}

desired both to ensure the provision of the least toxic and most effective therapy to the individual patient and for interrupting the transmission of drug resistant pathogens. For some drugs this is difficult to achieve with the standard, at most semi-quantitative culture methods currently in use.

Unfortunately DST for tuberculosis (TB) is too slow, complex or dangerous to be effectively implemented in all settings, therefore novel strategies with the potential to be automated warrant investigation [2]. We have previously described an in-house (myco) bacterial culture method [3], in which the growth of microcolonies from individual colony forming units (CFUs) is monitored similar to the 
ScanLag system [4]. But in addition, in our system the microcolonies can be transferred to different media whilst monitoring their growth. Thus, it is possible to determine the susceptibility of growing colonies to a drug by measuring the change in growth rate after exposure. As each individual colony is monitored, detection of multiple phenotypes, for example a mixed resistant and susceptible bacterial population (heteroresistance), is possible [3].

Detection of RIF susceptibility and resistance in Mycobacterium tuberculosis lab strains was previously achieved within 8 days after inoculation and only 24 hours after exposure to RIF [3]. Transferring intact growing colonies onto selective medium during their exponential growth phase avoids the second lag phase and thus is more rapid than classical subculturing selected colonies onto plates or into liquid medium containing antibiotics. Also, as individual microcolony growth rates can be accurately monitored, more subtle effects may be detected such as partial inhibition. This may aid the identification of clinically significant resistance in strains that (due to an MIC close to the breakpoint concentration) is more difficult to detect using traditional methods. Also the ability of the system to detect more subtle in vitro responses to antibiotic challenge may allow the detection of additional phenotypes with altered response to in vivo therapy. Therefore, we investigated the capacity of our microcolony approach for DST of a more challenging antibiotic Ethambutol (EMB).

Standard methods for DST are based on either the proportion method and performed on solid media, or on critical concentration assays performed in liquid culture systems [5]. More recently the observation of cording/ "microcolony" formation in liquid media in the Microscopic Observation Drug Susceptibility assay (MODS) has been applied [6,7].

For some antimycobacterial drugs such as isoniazid and RIF, the agreement between different methods and between different labs is very good, but for EMB, even in well-established labs susceptibility testing remains challenging $[8,9]$, possibly because the minimal inhibitory concentrations (MICs) of both wild type and EMB resistant strains are close to the recommended critical concentration [10]. Therefore maintaining reproducibility becomes more difficult than for antibiotics such as RIF, for which the majority of resistant strains have a MIC far above the breakpoint concentration [11,12].

Also, susceptibility testing against EMB does not correlate well with the known molecular markers of EMB resistance thus molecular testing produces results that are difficult to interpret. A number of mutations have been associated with EMB resistance, predominantly in embCAB $[13,14]$, but their association with resistance is not strong enough to classify strains as susceptible or resistant [14].
Here we investigate the performance of the microcolony method to differentiate between EMB resistant, intermediate and susceptible strains. Using four strains with known EMB susceptibility/resistance we determined microcolony growth classification criteria. Then the growth of microcolonies from a blinded panel of 20 TB strains was monitored and these strains classified using our classification criteria. Results were then compared to classical susceptibility testing performed in a reference laboratory.

\section{Methods}

\section{Strains}

A panel of 24 TB strains was received by the Royal Tropical Institute from the Swedish Institute for Communicable Disease Control (SMI) on Lowenstein Jensen slopes (Table 1). Strains were coded 1-24 and from strains $1-4$ the susceptibility testing results were provided to serve as reference. Strain 1 was labeled susceptible (S), strain 2 intermediate (I) and strains 3 and 4 resistant (R). No information was provided from strains 5-24 prior to communication of the results to the SMI.

All strains were inoculated in Middlebrook (MB) $7 \mathrm{H} 9$ medium (Difco, BD, Sparks, MD, USA) + OADC (BBL, BD) and subcultured every 3-4 weeks.

To control laboratory procedures multiplex ligationdependent probe amplification (MLPA) [15] was performed on DNA isolated from the $1^{\text {st }}$ routine cultures and from the last subcultures after testing of the panel, this yielded identical results for all (45) resistance associated markers and lineage type markers for all individual strains (data not shown).

\section{DST performed with BACTEC MGIT 960 and Middlebrook proportion method}

Strains were tested to 4 and $8 \mathrm{mg} / \mathrm{L}$ EMB (Sigma-Aldrich, Stockholm, Sweden) using the Middlebrook 7H10 agar proportion method as earlier described [10] or to 2.5 and $5 \mathrm{mg} / \mathrm{L}$ using the BACTEC MGIT 960 system (Becton Dickinson, Sparks, MD).

For the BACTEC MGIT, the DST inoculum was prepared from bacterial growth on Löwenstein-Jensen egg medium in $37^{\circ} \mathrm{C}$. Briefly, two $1 \mu \mathrm{L}$-loops of bacteria were suspended in $3 \mathrm{ml}$ of phosphate buffered saline (PBS) in a small glass tube with glass beads. Homogenization of the bacterial suspension was obtained by vortexing or in an ultrasound water bath to disperse clumps. Thereafter the suspension was left to sediment for $20 \mathrm{~min}$ and the upper $2 \mathrm{~mL}$ were transferred to a new tube and let to sediment for another $15 \mathrm{~min}$.

Prior to inoculation of the BACTEC MGIT culture tubes the bacterial suspension was adjusted to a McFarland turbidity of 0.5 and diluted in PBS according to the test protocol from the manufacturer. The drug susceptibility 
Table 1 Classification of the tested strains

\begin{tabular}{|c|c|c|c|c|}
\hline strain & SMI Strain number & Threshold concentration $(\mathrm{mg} / \mathrm{L}) \dagger$ & Microcolony growth-based classification (This study) & SMI classification \\
\hline 1 & XTB 10-172 & 1 & $\mathrm{~S}$ & $\mathrm{~S}$ \\
\hline 2 & XTB 09-045 & 4 & I & । \\
\hline 3 & XTB 09-023 & 16 & $\mathrm{R}$ & $\mathrm{R}$ \\
\hline 4 & XTB 09-060 & 16 & $\mathrm{R}$ & $\mathrm{R}$ \\
\hline 22\# & XTB 09-060 & $>8$ & $\mathrm{R}$ & R \\
\hline $24 \#$ & XTB 09-060 & $>8$ & $\mathrm{R}$ & $\mathrm{R}$ \\
\hline 5 & XTB 10-167 & $\leq 1$ & S & S \\
\hline 6 & XTB 10-166 & 4 & I & $S$ \\
\hline 7 & XTB 10-158 & $\leq 2$ & S & S \\
\hline 8 & XTB 09-109 & $>8$ & $R$ & $\mathrm{R}$ \\
\hline 9 & XTB 09-108 & 8 & $\mathrm{I} / \mathrm{R}^{*}$ & I \\
\hline 10 & ВТВ 09-554 & $>8$ & R & R \\
\hline 11 & BTB 11-250 & 4 & I & । \\
\hline 12 & BTB 11-214 & $>4$ & $\mathrm{R}$ & $\mathrm{R}$ \\
\hline 13 & BTB 11-145 & 2 & S & S \\
\hline 14 & BTB 11-421 & 2 & S & S \\
\hline 15 & XTB 10-170 & $>8$ & R & R \\
\hline 23\# & XTB 10-170 & $>4$ & $\mathrm{R}$ & $\mathrm{R}$ \\
\hline 16 & BTB 11-416 & 4 & I & $\mathrm{S} / \mathrm{l}$ \\
\hline 17 & BTB 11-417 & $\leq 1$ & S & S \\
\hline 18 & BTB 11-418 & $\leq 1$ & S & S \\
\hline 19 & BTB 11-419 & $\leq 1$ & S & S \\
\hline 20 & BTB 11-420 & $\leq 1$ & S & S \\
\hline 21 & ВТВ09-553 & $>4$ & R & R \\
\hline
\end{tabular}

Strains 1-4: reference strains, strains 5-24: blinded strains. † Threshold concentration, lowest concentration tested at which $>40 \%$ reduction in growth rate compared to control (no EMB) was recorded. Where $40 \%$ reduction in growth rate was reached at the lowest concentration tested a $\leq$ symbol precedes the concentration, Where the threshold was not reached at the highest concentration tested, this is indicated by a $>$ symbol. ${ }^{*} 4 \mathrm{mg} / \mathrm{L}$ missing due to contamination. When retested, strain 9 was correctly classified as intermediate. \# Strains 22 and 24, and strain 23 were (unknown) duplicates of strain 4 and 15 , respectively. The 2 results discordant between the microcolony growth-based classification and the SMI classifications are indicated in boldface.

to the recommended critical concentration of $5 \mathrm{mg} / \mathrm{L}$ EMB was assessed using the Becton Dickinson kit.

Strains were determined EMB susceptible when the growth unit (GU) of the culture tubes containing 2.5$5 \mathrm{mg} / \mathrm{L}$ EMB were $\leq 100$ when the 1:100 diluted drug free control had reached $G U=400$. Strains growing at concentrations between 2.5 and $5 \mathrm{mg} / \mathrm{L}$ were considered intermediary (I) resistant.

\section{Microcolony growth monitoring}

Microcolony growth was performed in 4-well culture plates (Nunc multidish 4. SI, Nunc A/S, Denmark), on squares of porous aluminium oxide (PAO, Whatman, Kent, UK) on top of MB7H10 agar (Difco, BD, Sparks, MD, USA) + OADC. Agar was prepared in standard petri dishes, and disks punched out of the agar were transferred to the 4-well culture plates. Strips of $3.6 \times 0.8 \mathrm{~cm}$ PAO were sterilized by submerging in $100 \%$ ethanol and cut into 4 squares of approx. $0.8 \times 0.8 \mathrm{~cm}$. After evaporation of the ethanol PAO squares were wetted in sterile water and placed on MB7H10 + OADC agar in the 4-well culture plates.

Mycobacterial cultures were vortexed for 20s and sedimented for at least $15 \mathrm{~min}$. A $2 \mathrm{ml}$ aliquot of the suspension was filtered through a $5 \mu \mathrm{m}$ filter (Whatman) to obtain a suspension of single cells. Cells were diluted in MB7H9 to approx. $3 \times 10^{6}$ cells $/ \mathrm{ml}$ based on the assumption that an $\mathrm{OD}_{450}$ of 0.15 corresponds to $10^{8}$ cells $/ \mathrm{ml}$. Three $\mu \mathrm{l}$ of this suspension was inoculated on the squares of PAO. Plates were incubated at $36^{\circ} \mathrm{C}$.

A stock solution of $4 \mathrm{mg} / \mathrm{ml} \mathrm{EMB} \mathrm{in} \mathrm{water} \mathrm{was} \mathrm{pre-}$ pared and stored aliquoted at $-20^{\circ} \mathrm{C}$. Four-well plates with MB7H10 agar + OADC supplemented with different concentrations of EMB (Ethambutol dihydrochloride, Sigma-Aldrich, St. Louis, MO, USA) were prepared as described above and used within 9 days.

Initially, growth of all 24 strains on non selective medium was monitored between days 5 and 16 or until the colonies covered the surface to determine the time 
frame for the EMB exposure experiments. Based on the results of experiments, exposure experiments to EMB were set up as follows:

For the four reference strains 1-4 eight squares of PAO were inoculated and grown for 7 days on nonselective medium. The PAO squares containing microcolonies were manually transferred to $0 ; 0.5 ; 1 ; 2 ; 4 ; 5 ; 8$ and $16 \mu \mathrm{g} / \mathrm{ml} \mathrm{EMB}$. Using an automated microscope system (Muscan, CCM BV, Nuenen, The Netherlands), images were recorded at 5 and 7 days after inoculation (pre-exposure baseline), directly after transfer (exposure to EMB began 7 days after inoculation) and then after 2, 5, 7 and 9 days of EMB exposure (corresponding with days $7,9,12,14$ and 16 after inoculation). Imaging was terminated at 16 days or if single colonies could no longer be discriminated due to overgrowth.

Based on the results of the four reference strains 1-4 (Figures 1 and 2) it was decided to test the blinded panel using the same conditions and transfer on day 7 , except that exposures were limited to concentrations of $0 ; 1 ; 2$; 4 and $8 \mu \mathrm{g} / \mathrm{ml} \mathrm{EMB}$. Imaging was performed at the same time points as above.

\section{Image and data analysis}

Image analysis was performed essentially as described in den Hertog et al. [3], but using a second generation microscope system (Muscan) allowing for higher throughput, and another software package with additional tools (FIJI; [16]).

Microcolony microscopy was performed using an automated microscopy system (Muscan) with bright field imaging, coaxial illumination, and a $5 \times$ objective. Images were recorded with a monochrome CCD camera (XCD-SX90, Sony, Tokio, Japan; with field size 1280×960 pixels). From each well at each time point a set of 81 images ( 9 by 9 images of $0.95 \times 0.71 \mathrm{~mm}$ each with an overlap of approx. $5.6 \%$ and $16.2 \%$ in $\mathrm{X}$ and $\mathrm{Y}$ direction respectively) was collected covering a total area of approx. $8.0 \times 5.5 \mathrm{~mm}$.

Using FIJI, first the set of 81 photos recorded per PAO square were stitched into a single image using the "Stitching 2D/3D" plugin [17]. Next, all stitched TIF images of the same PAO taken at the different time points were stacked and registered using the "Linear stack alignment with SIFT" plugin [18] to align potential colonies over all time points. Standard settings were used

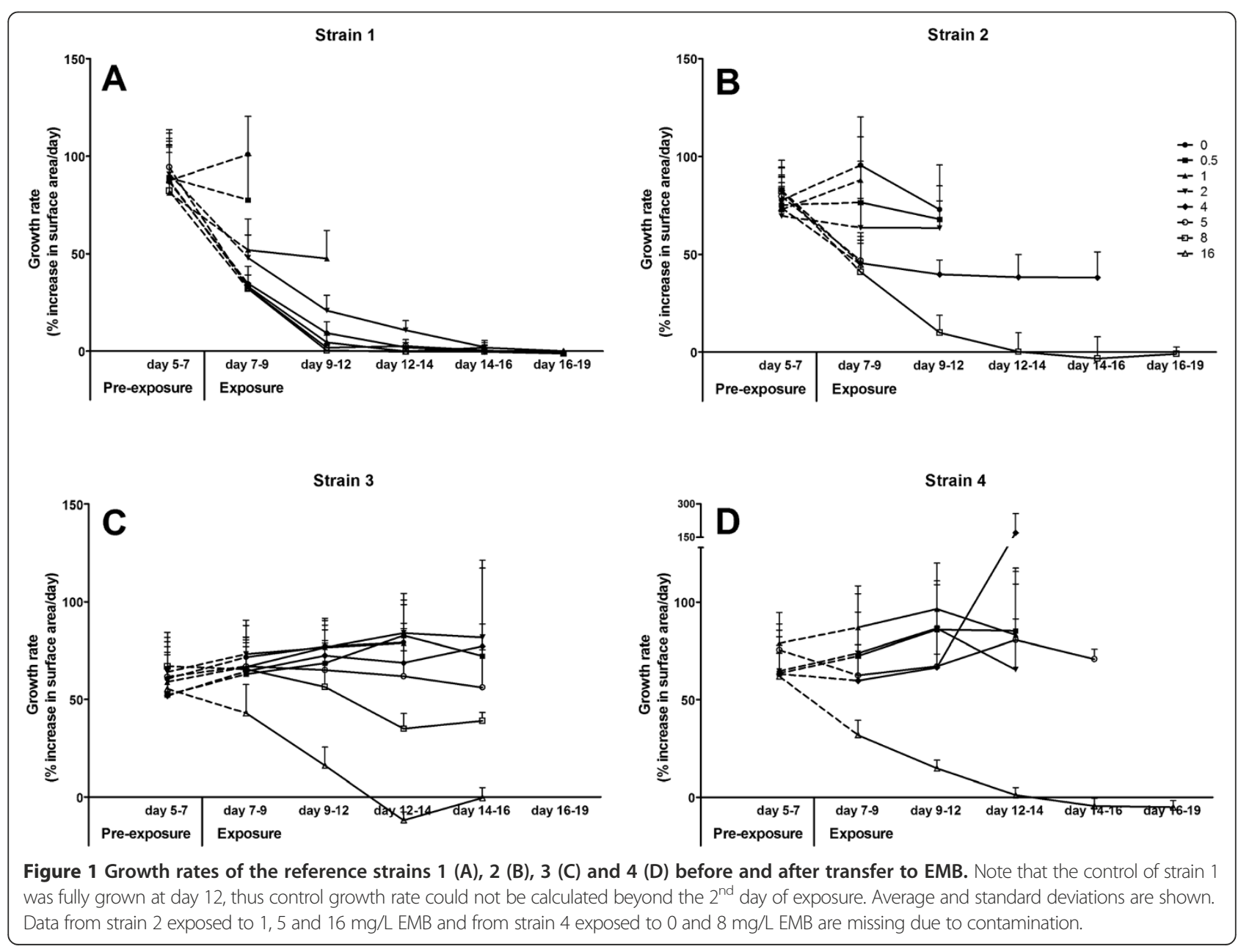




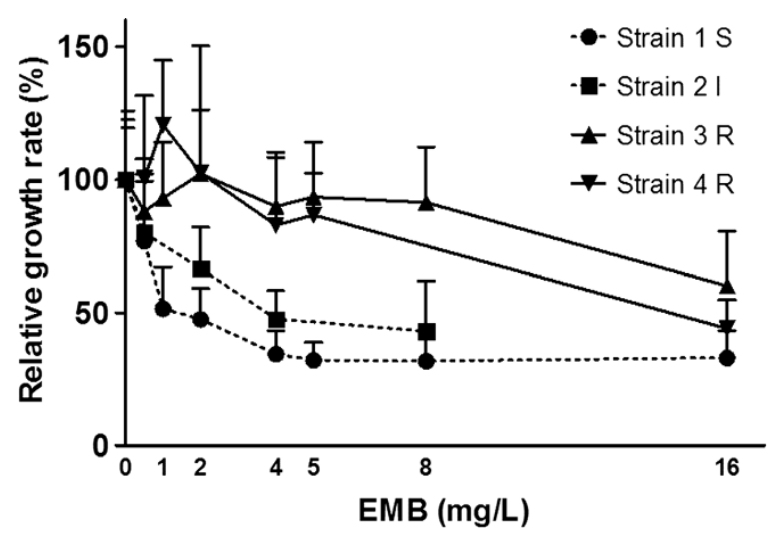

Figure 2 Relative growth rates of reference strains (as \% of $0 \mathrm{mg} / \mathrm{L}$ control) between days 7 and 9 after transfer to EMB on day 7.

*For strain 4 results are shown relative to the pre-exposure growth rate due to contamination of the control. Error bars represent the standard deviation of the relative growth rate of individual microcolonies.

except the minimum and maximum image size for the scale invariant Interest point detector were set at 200 and 3000 respectively.

Macros were written to perform the following steps in FIJI: first a background reduction (with rolling ball radius of 200 pixels) and a 2 pixel radius median filter were applied. Next, using the "RenyiEntropy" method the images were converted to binary with a threshold level of 238. Using the "Analyse particles" tool the sizes and $\mathrm{XY}$ coordinates of all particles with a minimal size threshold of 50 pixels and a circularity of or above 0.5 were saved into a text file.

The data extracted from the images was then processed using the web application described by den Hertog et al. [3] to identify particles with equivalent $\mathrm{XY}$ coordinates present in at least two sequential time points. The sizes of the resulting lists of objects at sequential time points were exported to excel for further analysis. On basis of this information individual colony growth rates, defined as \% increase in surface area/day, were calculated for all strains and conditions between sequential time points.

Objects that fulfilled the following were identified as mycobacterial colonies:

- An increase in surface area of the object of $>20 \%$ per day between days 5 and 7 after inoculation.

- No significant effect of transfer on the objects surface area $(<20 \%$ change); i.e. similar surface area of both measurements on day 7 after inoculation made before and after transfer

- Presence and no decrease in size $(<10 \%$ per day) of the object after transfer to selective medium (or control) days 7 and 9.

\section{Classification}

Based on the growth inhibition profiles of the reference strains 1-4 (See Results section), criteria were defined to allow the unknown strains to be classified. At each concentration of EMB the relative average growth rate compared to the unexposed control (0 $\mathrm{mg} / \mathrm{L} \mathrm{EMB})$ was calculated for days 7-9, 0 to 2 days EMB exposure. Strains were classified based on the lowest EMB concentration resulting in a decrease in relative growth rate (compared to the unexposed control) of $>40 \%$; If growth was decreased $40 \%$ or more at i. concentrations $\leq 2 \mathrm{mg} / \mathrm{L}$, strains were classified as Susceptible, ii. $4 \mathrm{mg} / \mathrm{L}$ as Intermediate, iii. if growth rate was reduced less than $40 \%$ at $>4 \mathrm{mg} / \mathrm{L}$ as Resistant.

\section{Results}

Baseline growth rate of strains on non selective medium Growth of all strains was monitored from 5 days to 16 days after inoculation or until confluence was reached. The data collected was used to determine for the EMB exposure experiments at which time points to measure the baseline and transfer the microcolonies to EMB. All strains had stable growth rates measured between days 5-9 and when data was available, between days 5-12. All strains were thus in the exponential growth phase between days 5 and 9 . Based on data from 629 to 9608 (median 3911.5) microcolonies per strain the average microcolony surface area between days 5 and 7 after inoculation increased by $45.6 \%$ to $114.7 \%$ per day (Figure 3). These growth rates correspond to doubling times of 20.9 to 52.6 hours.

Despite the wide range in growth rates, microcolonies from all strains were detectable 5 days after inoculation. Therefore, imaging was begun at day 5 and exposure to EMB at day 7.

\section{Effects of EMB on the growth of reference strains}

To establish criteria to classify the blinded strains, four reference strains with known EMB susceptibility (susceptible, intermediate and resistant) were exposed to $0-16 \mathrm{mg} / \mathrm{L}$ 


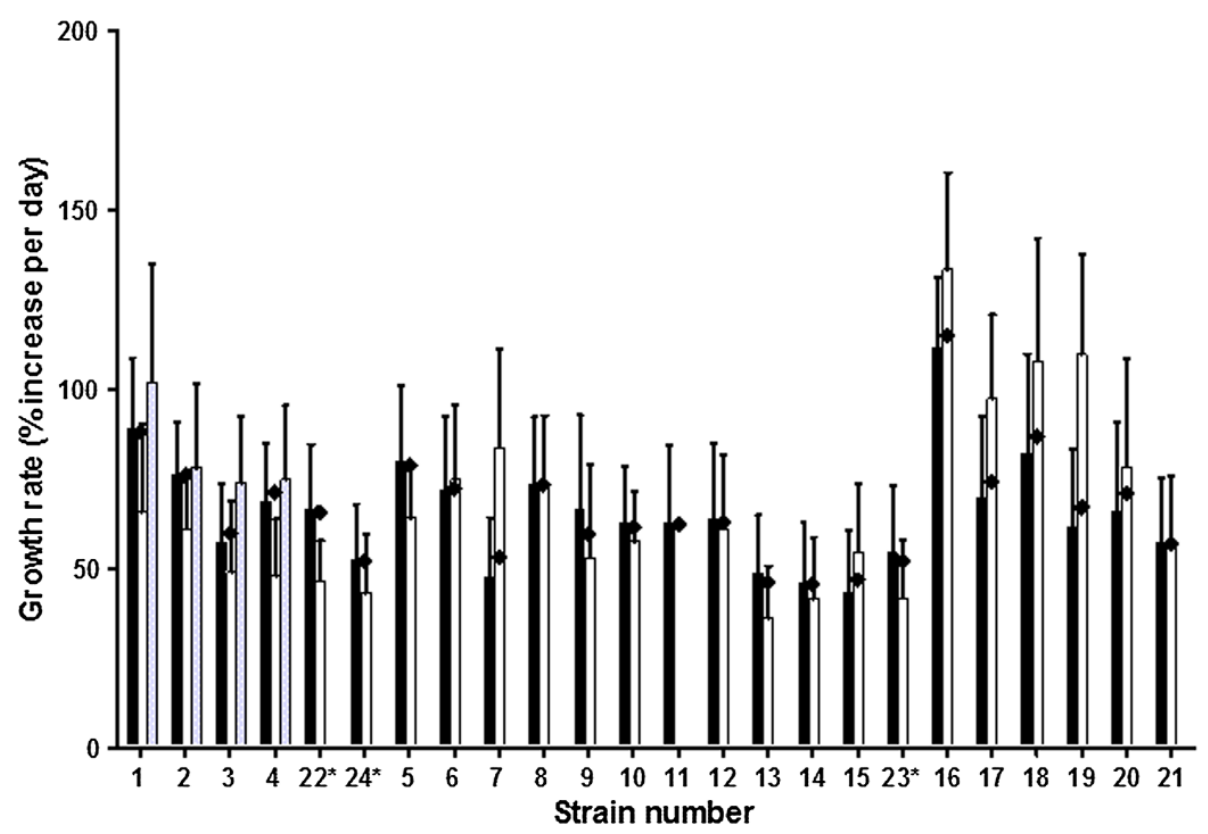

Figure 3 Growth rates (\% increase in surface area per day) of strains 1-24 between days 5 and 7 after inoculation. Pre-exposure data from the EMB exposure experiments (black bars), and data collected in the baseline experiments (white and grey bars) are combined. Diamonds represent the weighted average of all data points for each strain, ranging between 629 and 9608 (median 3911.5) data points per strain. *As shown in Table 1, strains 22 and 24 are the same strain as strain 4, and strain 23 is the same as strain 15, although they were included as individual strains in the blinded panel. Error bars represent the standard deviation of the growth rate of individual microcolonies.

EMB from day 7 after inoculation and growth curves for individual microcolonies were produced (Figure 1).

The growth rate of the susceptible strain 1 was approximately halved by the presence of $1 \mathrm{mg} / \mathrm{L} \mathrm{EMB}$ within the $1^{\text {st }} 2$ days of exposure (days 7-9). After day 9 (2 days EMB exposure) the growth of strain 1 was completely inhibited by concentrations higher than $4 \mathrm{mg} / \mathrm{L}$ (Figure 1A). In contrast, the growth rate of intermediate resistant strain 2 was unaffected by the presence of up to $2 \mathrm{mg} / \mathrm{L}$ EMB for the $1^{\text {st }} 5$ days of exposure (day 12) but clear inhibition was seen for concentrations over $4 \mathrm{mg} / \mathrm{L}$ EMB from day 7 onwards (Figure 1A). The growth rate of resistant strains 3 and 4 was only reduced by $16 \mathrm{mg} / \mathrm{L}$ of EMB when compared to the antibiotic free control or the pre-exposure growth rate (Figure $1 C$-D).

The data collected between days 7 and 9 presented in Figure 1 was used to calculate the relative growth rates compared to the unexposed controls $(0 \mathrm{mg} / \mathrm{L}$ for all EMB concentrations) for each strain (Figure 2). Based on this analysis it was decided to expose the strains in the blinded panel to $0 ; 1 ; 2 ; 4$ and $8 \mathrm{mg} / \mathrm{L}$ EMB. Furthermore a classification scheme was established as follows: Strains with a decrease in growth rate compared to the unexposed control between 0-2 days of exposure of more than $40 \%$ at $<2 \mathrm{mg} / \mathrm{L}$ were classified as susceptible, a decrease in growth rate of $>40 \%$ between $2-4 \mathrm{mg} / \mathrm{L}$ as intermediate, and a decrease in growth rate of $<40 \%$ at
$4 \mathrm{mg} / \mathrm{L}$ as resistant. The resulting classification was then reported to the reference laboratory and only then the code broken.

Using the microcolony method eight strains were classified as susceptible (Figure 4A, Table 1), three strains as intermediate (Figure $4 \mathrm{~B}$ ) and eight strains as resistant (Figure 4C). Classification of strain 9 was uncertain (between intermediate and resistant Figure 4B-C) due to missing data, contamination of the $4 \mathrm{mg} / \mathrm{L} \mathrm{PAO}$ filter. Upon breaking the code and comparing to the classification and MICs of the strains as determined by the Swedish Institute for Communicable Disease Control (Table 1) all resistant strains (strains 8, 10, 12, $15,21,22,23$ and 24) were found to have been correctly identified. Classification of the remaining strains all with a MICs below the breakpoint concentration of $5 \mathrm{mg} / \mathrm{L}$ was in agreement (intermediate or susceptible) by both methods except for strain 6 which was identified as intermediate by the microcolony method and susceptible by the reference laboratory (Table 1) and strain 9 which was not grouped due to missing data. Retesting of strain 9 after breaking the code resulted in corresponding classification as intermediate (data not shown).

Frequency distributions of the relative growth rates between days 7-9 of the exposed microcolonies showed homogeneous responses in all conditions for every strain (Additional file 1). 


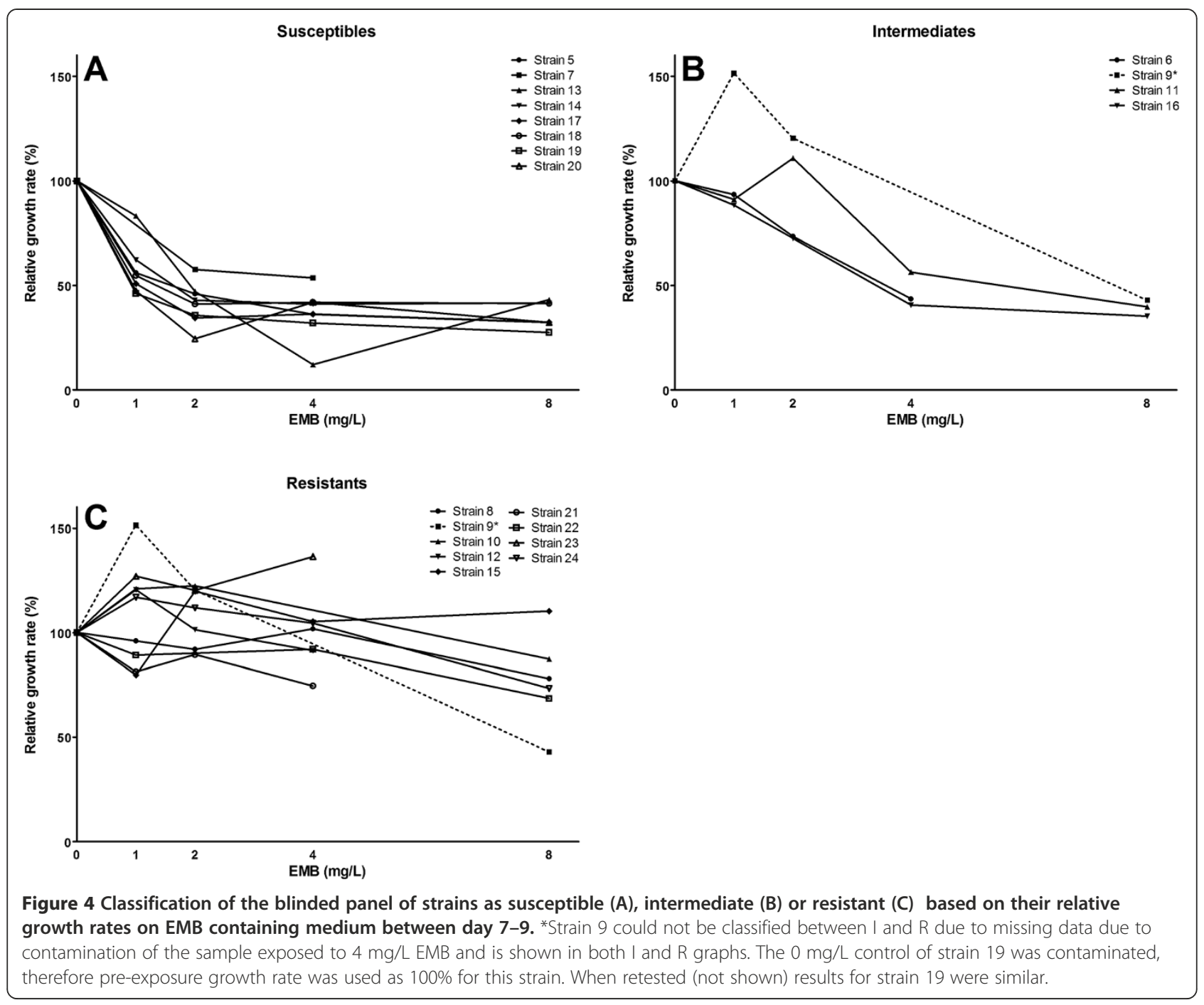

\section{Discussion}

EMB susceptibility testing of $M$. tuberculosis isolates is challenging and difficult to perform reproducibly [19]. Here we have shown that with our microcolony growth monitoring method exposure of growing microcolonies to EMB can provide clear and accurate EMB susceptibility determinations within 2 days of exposure (9 days after initial inoculation). In this study the growth of a total of more than 100,000 automatically identified microcolonies was tracked.

A blinded panel of 20 strains was tested, 18 of which were classified by our method into identical groups as the reference laboratory using the gold standard method. One strain (strain 6) classified by our method as intermediate was classified as susceptible by the reference laboratory. Another strain (strain 9) could not be classified more accurately than "Intermediate or Resistant" from the initial blinded testing due to laboratory contamination of the $4 \mathrm{mg} / \mathrm{L}$ exposure (Figure 4), although when retested after breaking the code it was correctly classified as Intermediate by the microcolony method (not shown).

We classified the strains on the basis of \% growth inhibition compared to an antibiotic free control at 2 concentrations ( 2 and $4 \mathrm{mg} / \mathrm{L}$ of EMB). However our method results in an unprecedented amount of data and it may be possible, if growth conditions are carefully selected, to determine EMB susceptibility by testing at single time point with only a single concentration. For example, after breaking the code we realized that the relative growth rate after 48 hours on $2 \mathrm{mg} / \mathrm{L}$ alone would have been sufficient to classify 17 out of 20 strains identically to the reference method (by classifying strains as sensitive strains with a relative growth rate $<60 \%$, strains as intermediate with a growth rate between 60 and $80 \%$, and strains as resistant with a growth rate of $>80 \%$ of the control (Figure 4)). Also in this study we decided to classify after 2 days of EMB exposure but findings of de Steenwinkel et al. [20] demonstrate that EMB inhibition in liquid cultures is detectable 
within 24 Hours. As our method can also detect subtle changes in growth we believe classification may have been possible after only 1 days exposure to EMB.

A number of mutations, predominantly in the $e m b B$ gene, associated with EMB resistance have been identified, but the correlation with susceptibility testing is low [21,22] making the clinical significance of the mutations based on classical phenotypic testing results difficult to determine [23]. In a recent study [24], the spectrum of EMB resistance associated mutations has been expanded with other genes involved in decaprenylphosphoryl- $\beta$ - $D$-arabinose (DPA) biosynthetic and utilization pathways both in vitro and in vivo. The authors demonstrate that resistance is acquired or increased step-wise, and multiple mutations in different genes including $e m b C A B$ are required to obtain high level resistance. The broad spectrum of mutations that diversely affect the susceptibility explains the difficulty of phenotypic testing for EMB when compared to some other antibiotics for which the number of (identified) relevant resistance conferring mutations are limited and their effect on the MIC is dramatic. This results in poorer agreement between DST from different labs for EMB compared to other antibiotics such as RIF and isoniazid $[10,19]$.

We have shown that inhibition of growth is not always complete, which would be an additional complication for traditional DST assays that can yield a positive or negative result at each concentration. For instance, the intermediately susceptible strain 2 continues to grow for at least 10 days with a constantly reduced growth rate of approx. $50 \%$ on $4 \mathrm{mg} / \mathrm{L}$ EMB (Figure 1). This partial inhibition was confirmed to be due to reduced growth rate of all colonies and thus is not an artifact due to heteroresistance (eg. a mixed genotype), which would have been detected as a proportion of colonies growing at the normal rate and a proportion completely inhibited (not shown, except for days 7-9 in Additional file 1).

The large numbers of mutations involved in EMB resistance make the development of accurate molecular assays for detecting resistance associated mutations as proxy for phenotypic susceptibility a huge challenge. In order to model the subtle effects of the different mutations on susceptibility to EMB, accurate measurement of the inhibition of growth rate of a statistically robust number of colonies in the presence of EMB may be more informative than only the MIC value resulting from traditional culture methods.

For this study, our data analysis treated all colonies from a single exposure as a homogeneous population, which was confirmed to be true based on the frequency distributions of growth rates between days 7-9 (Additional file 1). However, as the number of colonies analyzed per condition is large (generally $>100$ ), and analysis based on the frequency distributions of growth rates would be a powerful tool for detecting heteroresistance or confirming a single phenotype as was the case in this study.
Presently our method requires the careful manipulation of the PAO filters containing growing microcolonies and a simple device to facilitate this step will be required before the method can be widely adopted. Current we and our partners are working on the development of such a device as well as integrating our image analysis method into a user friendly microscopic reader with integrated software in order to make a system available to other researchers and more suitable to high throughput applications.

\section{Conclusion}

In conclusion, monitoring of multiple microcolonies allows rapid determination of EMB susceptibility and has applications in diagnostics and for characterization of the inhibitory effect of EMB on strains carrying resistance associated mutations.

\section{Additional file}

Additional file 1: Frequency distributions of relative growth rates of strains 1-24 between days 7-9. Frequency distributions of the growth rate relative to the averaged unexposed $0 \mathrm{mg} / \mathrm{L}$ control are shown for each strain. Distributions are shown with $20 \%$ bins, and data are plotted at the upper limit of each bin. (e.g. data over $80-100 \%$ is plotted at 100\%). Frequency is expressed as the \% of total counts.

\section{Abbreviations}

CFU: Colony forming unit; DST: Drug susceptibility testing; EMB: Ethambutol; GU: Growth unit; I: Intermediate; MIC: Minimal Inhibitory concentration; MLPA: Multiplex ligation-dependent probe amplification; PAO: Porous aluminum oxide; PBS: Phosphate Buffered saline; R: Resistant; RIF: Rifampicin; S: Susceptible; TB: Tuberculosis.

\section{Competing interests}

The Royal Tropical Institute has a financial stake in the culture system and has a patent describing the culture system.

\section{Authors' contributions}

$\mathrm{AH}$ and RA designed the study, defined classification criteria and drafted the manuscript. SM and ES carried out the experiments. ES and AH performed the data analysis. JW and SH set up the strain panel, provided the reference results and helped draft the manuscript. All authors read and approved the final manuscript.

\section{Acknowledgements}

We would like to thank Anja Schuitema for performing the MLPA. We acknowledge NanonextNL for funding enabling this research.

\section{Author details}

${ }^{1}$ Royal Tropical Institute, KIT Biomedical Research, Meibergdreef 39, 1105, AZ, Amsterdam, The Netherlands. ${ }^{2}$ Department of Diagnostics and Vaccines, Unit of Highly Pathogenic Microorganisms, Swedish Institute for Communicable Disease Control, S-171 82, Solna, Sweden.

Received: 2 December 2013 Accepted: 3 July 2014

Published: 10 July 2014

\section{References}

1. Boehme CC, Nabeta P, Hillemann D, Nicol MP, Shenai S, Krapp F, Allen J, Tahirli R, Blakemore R, Rustomjee R, Milovic A, Jones M, O'Brien SM, Persing DH, Ruesch-Gerdes S, Gotuzzo E, Rodrigues C, Alland D, Perkins MD: Rapid molecular detection of tuberculosis and rifampin resistance. $N$ Engl J Med 2010, 363(11):1005-1015 
2. Wells WA, Boehme CC, Cobelens FG, Daniels C, Dowdy D, Gardiner E, Gheuens J, Kim P, Kimerling ME, Kreiswirth B, Lienhardt C, Mdluli K, Pai M, Perkins MD, Peter T, Zignol M, Zumla A, Schito M: Alignment of new tuberculosis drug regimens and drug susceptibility testing: a framework for action. Lancet Infect Dis 2013, 13(5):449-458.

3. den Hertog AL, Visser DW, Ingham CJ, Fey FH, Klatser PR, Anthony RM: Simplified automated image analysis for detection and phenotyping of Mycobacterium tuberculosis on porous supports by monitoring growing microcolonies. PLOS ONE 2010, 5(6):e11008.

4. Levin-Reisman I, Gefen O, Fridman O, Ronin I, Shwa D, Sheftel H, Balaban NQ: Automated imaging with ScanLag reveals previously undetectable bacterial growth phenotypes. Nat Methods 2010, 7(9):737-739.

5. Drobniewski F, Rüsch-Gerdes S, Hoffner S: Subcommittee on Antimicrobial Susceptibility Testing of Mycobacterium tuberculosis of the European Committee for Antimicrobial Susceptibility Testing (EUCAST) of the European Society of Clinical Microbiology and Infectious Diseases (ESCMID). Antimicrobial susceptibility testing of Mycobacterium tuberculosis (EUCAST document E.DEF 8.1): report of the Subcommittee on Antimicrobial Susceptibility Testing of Mycobacterium tuberculosis of the European Committee for Antimicrobial Susceptibility Testing (EUCAST) of the European Society of Clinical Microbiology and Infectious Diseases (ESCMID). Clin Microbiol Infect 2007, 13(12):1144-1156.

6. Mendoza A, Castillo E, Gamarra N, Huamán T, Perea M, Monroi Y, Salazar R, Coronel J, Acurio M, Obregón G, Roper M, Bonilla C, Asencios L, Moore DA Reliability of the MODS assay decentralisation process in three health regions in Peru. Int J Tuberc Lung Dis 2011, 15(2):217-222.

7. Zimic M, Velazco A, Comina G, Coronel J, Fuentes P, Luna CG, Sheen P, Gilman RH, Moore DA: Development of low-Cost inverted microscope to detect early growth of Mycobacterium tuberculosis in MODS Culture. PLOS ONE 2010, 5(3):e9577.

8. Van Deun A, Wright A, Zignol M, Weyer K, Rieder HL: Drug susceptibility testing proficiency in the network of supranational tuberculosis reference laboratories. Int J Tuberc Lung Dis 2011, 15(1):116-124.

9. Madison B, Robinson-Dunn B, George I, Gross W, Lipman H, Metchock B, Sloutsky A, Washabaugh G, Mazurek G, Ridderhof J: Multicenter evaluation of ethambutol susceptibility testing of Mycobacterium tuberculosis by agar proportion and radiometric methods. J Clin Microbiol 2002, 40(11):3976-3979.

10. Schön T, Juréen P, Giske CG, Chryssanthou E, Sturegård E, Werngren J, Kahlmeter G, Hoffner SE, Angeby KA: Evaluation of wild-type MIC distributions as a tool for determination of clinical breakpoints for Mycobacterium tuberculosis. J Antimicrob Chemother 2009, 64(4):786-793.

11. Huitric E, Werngren J, Juréen $P$, Hoffner S: Resistance levels and $r p o B$ gene mutations among in vitro-selected rifampin-resistant Mycobacterium tuberculosis mutants. Antimicrob Agents Chemother 2006, 50(8):2860-2862

12. Bergval IL, Klatser PR, Schuitema AR, Oskam L, Anthony RM: Specific mutations in the Mycobacterium tuberculosis $r p o B$ gene are associated with increased dnaE2 expression. FEMS Microbiol Lett 2007, 275(2):338-343

13. Telenti A, Philipp WJ, Sreevatsan S, Bernasconi C, Stockbauer KE, Wieles B, Musser JM, Jacobs WR Jr: The emb operon, a gene cluster of Mycobacterium tuberculosis involved in resistance to ethambutol. Nat Med 1997, 3(5):567-570.

14. Engström A, Morcillo N, Imperiale B, Hoffner SE, Juréen P: Detection of first- and second-line drug resistance in Mycobacterium tuberculosis clinical isolates by pyrosequencing. J Clin Microbio/ 2012, 50(6):2026-2033.

15. Bergval I, Sengstake S, Brankova N, Levterova V, Abadía E, Tadumaze N, Bablishvili N, Akhalaia M, Tuin K, Schuitema A, Panaiotov S, Bachiyska E, Kantardjiev T, de Zwaan R, Schürch A, Van Soolingen D, Van't Hoog A, Cobelens F, Aspindzelashvili R, Sola C, Klatser P, Anthony R: Combined species identification, genotyping, and drug resistance detection of Mycobacterium tuberculosis cultures by MLPA on a bead-based array. PLOS ONE 2012, 7(8):e43240.

16. Schindelin J, Arganda-Carreras I, Frise E, Kaynig V, Longair M, Pietzsch T, Preibisch S, Rueden C, Saalfeld S, Schmid B, Tinevez JY, White DJ, Hartenstein V, Eliceiri K, Tomancak P, Cardona A: Fiji: an open-source platform for biological-image analysis. Nat Methods 2012, 9(7):676-682.

17. Preibisch S, Saalfeld S, Tomancak P: Globally optimal stitching of tiled $3 D$ microscopic image acquisitions. Bioinformatics 2009, 25(11):1463-1465.

18. Lowe DG: Distinctive Image Features from Scale-Invariant Keypoints. Int J Comput Vision 2004, 60(2):91-110.
19. Horne DJ, Pinto LM, Arentz M, Lin SY, Desmond E, Flores LL, Steingart KR, Minion J: Diagnostic accuracy and reproducibility of WHO-endorsed phenotypic drug susceptibility testing methods for first-line and second-line antituberculosis drugs. J Clin Microbiol 2013, 51(2):393-401.

20. de Steenwinkel JE, de Knegt GJ, ten Kate MT, van Belkum A, Verbrugh HA, Kremer K, van Soolingen D, Bakker-Woudenberg IA: Time-kill kinetics of anti-tuberculosis drugs, and emergence of resistance, in relation to metabolic activity of Mycobacterium tuberculosis. J Antimicrob Chemother 2010, 65(12):2582-2589.

21. Plinke C, Rüsch-Gerdes S, Niemann S: Significance of mutations in embB codon 306 for prediction of ethambutol resistance in clinical Mycobacterium tuberculosis Isolates. Antimicrob Agents Chemother 2006, 50(5):1900-1902.

22. Sirgel FA, Warren RM, Streicher EM, Victor TC, van Helden PD, Böttger EC embB306 mutations as molecular indicators to predict ethambutol susceptibility in Mycobacterium tuberculosis. Chemotherapy 2012, 58(5):358-363.

23. Feng Y, Liu S, Wang Q, Wang L, Tang S, Wang J, Lu W: Rapid diagnosis of drug resistance to fluoroquinolones, amikacin, capreomycin, kanamycin and ethambutol using genotype MTBDRsl assay: a meta-analysis. PLOS ONE 2013, 8(2):e55292.

24. Safi H, Lingaraju S, Amin A, Kim S, Jones M, Holmes M, McNeil M, Peterson SN, Chatterjee D, Fleischmann R, Alland D: Evolution of high-level ethambutol-resistant tuberculosis through interacting mutations in decaprenylphosphoryl- $\beta$-D-arabinose biosynthetic and utilization pathway genes. Nat Genet 2013, 45(10):1190-1197.

doi:10.1186/1471-2334-14-380

Cite this article as: den Hertog et al:: Evaluation of a microcolony growth monitoring method for the rapid determination of ethambutol resistance in Mycobacterium tuberculosis. BMC Infectious Diseases $201414: 380$

\section{Submit your next manuscript to BioMed Central and take full advantage of:}

- Convenient online submission

- Thorough peer review

- No space constraints or color figure charges

- Immediate publication on acceptance

- Inclusion in PubMed, CAS, Scopus and Google Scholar

- Research which is freely available for redistribution 